\title{
Where next is science heading?
}

The immediate future for science is bright, but the future may be clouded by poor public education and by too much competitiveness - the gist of a talk at Los Alamos on Tuesday.

\section{Santa Fe}

NATURALLY there is no way of telling where science is heading except in the most trivial sense. For example, one can safely guess that the new electron-positron accelerator (LEP) at CERN in Geneva, which will start operating a few weeks from now, will either discover the top quark, or it will not. In the first case, we shall all be a little more content. Otherwise we shall feel challenged and will start telling our governments that they should spend even more on high-energy physics than has been their custom for the past 40 years. Similarly, there are dozens of engineered biochemicals waiting in the wings for development to be complete and for the regulatory authorities to give their approval; if one of these should, for example, make good the defect we call schizophrenia, the world we live in would be different. So it will be when the pocketsized mainframe computer is for sale.

The outstanding feature of those developments is that they lie within the reach of the development process as we know it. The top quark is simply an entity which, by evil chance, has not yet been discovered. To suppose that genetic engineering will yield a chemical that cures schizophrenia is an exaggeration born of wishful thinking; as things are, nobody knows whether there is an identifiable biochemical defect underlying the disease, when there are only the sketchiest notions of what it might be and when the aetiology of the disease is so poorly understood that nobody can be sure whether the defect is reversible. But the chance that these uncertainties might be resolved, the social importance of this and a host of other natural diseases is so great and the search for a better understanding of them is intellectually so interesting that the programmes of biomedical research now supported by our governments are self-evidently justified. Indeed, that is why some people argue that they are too small.

The interesting questions about the more distant direction of science cannot be as simply answered. Of course, the momentum of present developments will, to some extent, condition the fields in which discoveries are made. One obvious illustration is the present interest in the human genome. The immediate goal of producing a nucleotide sequence is justified by the potential benefits of being able simply to look up in a library genes and their control elements which are found, by means of what will soon be straightforward biomedical research, to be involved in particular human diseases. But only a little reflection will show that long before the nucleotide map of the human genome is complete, human anthropologists will have recognized that these techniques provide them with a powerful new technique for understanding how the human population became what it is. More generally, it is only natural that the present concentration of talented people's energy in molecular biology will yield other benefits. We shall learn what is the genetic basis of speciation. We shall no doubt learn enough about the interactions of biomolecules to be able to simulate the origin of life. And, no doubt, molecular biology will soon move beyond its present preoccupation with the naming of the molecular parts of which organisms are made to a search for the dynamic relationships between them.

Similar prospectuses can easily be drawn up in other contexts. It is also clear that, despite the concentration of curiosity on the mechanism by which the outer skin of the Earth is kept habitable that has been growing since the International Geophysical Year, much more will now be learned not just about the stability of the biosphere, but about its manipulation. At least in principle, the discovery that anthopgenic emissions can affect the ozone layer works both ways: anthropogenic manipulation might be devised to protect it.

What the record of the past 40 years has shown is that the process of development has now become deliberate. it is possible to set goals and, within reason, to achieve them. That is what the construction of the first nuclear weapons showed. Since then, of course, we have also learnt, often painfully, that the achievement of a technical goal does not ensure that the products of development will be economically useful. Controlled thermonuclear fusion is an obvious case in which the technical feasibility of the process seems with difficulty assured, but the economics of the process are still an open question. Cold fusion, of course, is something else again.

But what lies beyond this predictable and mostly cheerful prospect for the next few decades? The simple answer is that there is no way of telling, given that the more distant future will hang on discoveries not yet made. but that presupposes that even the most important discoveries are accidental But that is an over-simple view. Take relativity for example.

It is in no way slighting of Einstein's reputation to assert that if he had not put forward the special theory of relatively in 1905 , somebody else would have done so in a very short time. In that case, we even know who would have done so. $\mathrm{H}$. A. Lorentz is one candidate, Poincaré so obviously another that there have been 80 years of vigorous argument on the question whether Poincare anticipated Einstein. The obvious parallel, now, is the continuing search for a unified theory of fundamental forces. When, quite soon perhaps, there is a theory that really hangs together, it will seem a puzzle to us all that it took such a long time coming.

What that implies is that, even if it is not feasible to anticipate the process of discovery, it is at least possible to think of conditioning the scientific process to make it more fruitful of discovery. Since the Second World War, we have learned, or believe we have learned, a great deal in that direction. This is why we honour basic research - and why we are constantly engaged in the argument of the balance between basic research and its application.

Nobody can deny that the growth of science in the United States, and the greatest success that has flowed from it, stems from the commitment to basic research of successive administrations since the Second World War. It is remarkably enlightened that the Bush administration is seeking further increases of the budgets of agencies such as the National Science Foundation in a year when it should be bending all its energies on the reduction of the federal deficit. On the face of things, that augers well.

But sadly, there is another side of the coin. Research prospers by the talents of those who enter the profession, but the state of public education and the social goals of young people threaten to restrict the supply of indigenous talent. Similarly, the competitiveness of the US research process, with its practical requirement that academic scientists should almost exclusively rely on competitive grants for their support, which explains the productivity of US science, has also had deleterious consequences. The flow of publications may have been increased, but not the exchange of ideas. There is in present circumstances a danger that science may no longer have room for scholarly people who do not care for the rat-race. That could be disastrous.

John Maddox 